Objective Neonatal recommendations include medical followup, metabolic screening, vitamin $\mathrm{K}$, eye drops/ointment, and vaccinations. Most but not all parents follow these recommendations. This study aimed to characterise the non-compliant mothers in order to understand the reasons behind that noncompliance.

Study design All mothers who refused any routine treatment of their infant or who asked for early discharge of their infant were included. They filled-in a questionnaire on socioeconomic items, previous births and those children's health status, current pregnancy and delivery, and their beliefs.

Result Three non-compliant subgroups emerged according to the responses: Muslim mothers requesting early discharge to care for children at home, Jewish mothers requesting early discharge because they disliked hospital environment, Sabbath/holiday, and children at home, and Jewish mothers who rejected guidelines because they believed the treatments were unnecessary or possibly harmful to their infants.

Conclusion Several interventions to enhance maternal compliance to medical recommendations are proposed, including more education, better quality of patient-staff interaction, understanding and encouraging social support, and awareness of patient's beliefs and attitudes, as well as renovation of the maternity department's physical structure, to make the stay more pleasant (quieter, more privacy), and to also make it possible to properly celebrate the Sabbath as well as holidays on the ward itself. Regarding the Islamic and Jewish mothers who asked for early discharge because of the other children at home, maybe we should try to understand the exact reason for this - perhaps the father works far away from home and there is nobody else to guard these children; and then we may be able to help these families find a better solution.

\section{P0-0623 NEONATAL DISCHARGE PLANNING: CAN WE REDUCE UNSCHEDULED REVIEWS IN THE FIRST 6 WEEKS OF LIFE?}

${ }^{1}$ EB Forman, ${ }^{2}$ NÓ Catháin, ${ }^{1} \mathrm{~A}$ Sills, ${ }^{3} \mathrm{~S}$ Cronin, ${ }^{2} \mathrm{~N}$ Mc Callion. ${ }^{1}$ Neonatology, Children's University Hospital Temple Street, Dublin, Ireland; ${ }^{2}$ Neonatology, Rotunda Hospital, Dublin, Ireland; ${ }^{3}$ Trainee, General Practice, Dublin, Ireland

\subsection{6/archdischild-2014-307384.1265}

Discharge planning for the well newborn is often overlooked and is increasingly recognised as an important function of any neonatal unit. We analysed factors associated with presentation of a newborn ( $<6$ weeks) to a local PED, GP practice and maternity hospital over a 3 week period.

Aim The aim was to gather data that could inform policy on discharge planning. This includes education for parents on the services available to them and on normal newborn care.

Methods Data was collected from an electronic database in the case of the PED and the local GP. It was collected prospectively manually in the maternity hospital.

Results In total 276 patients under 6 weeks of age presented to the three services over the three week period. Almost half of these visits were unscheduled (137/49\%). Of those that were unscheduled, 29\% were felt to represent benign neonatal variants whilst $20 \%$ were simple feeding problems. Discharge rates did not vary hugely between referrals made from GPs (56\%), Nurses (57\%) or the parents themselves (67\%).

Discussion A proportion of reviews were felt to be inappropriate and could have been prevented with parental education. The similar rates of discharge between the three sources of referral also suggests that both GPs and nurses in the community are not comfortable with caring for newborns. There is room for cost saving and quality improvement of the service through education.

\section{PO-0624 THE COST OF DISABILITY AND IMPROVING THE CONDITION OF NEONATES WITH HYPOXIC AND ISCHAEMIC IMPAIRMENT OF THE CENTRAL NERVOUS SYSTEM}

N Gamakova. Neonatology, Regional Hospital of Ruse Bulgaria, Ruse, Bulgaria

\subsection{6/archdischild-2014-307384.1266}

Background and aims Caring for a child with disability can be expensive not only for the family itself but also to society. Premature neonates are more likely to be re-hospitalised than babies born at full term and the healthcare costs in the first year are significantly higher for premature babies.

The cost of caring for a disabled child is clearly immense. As a result it is important to try to find ways to prevent disabilities or improve the condition of neonates who are at a risk of developing disabilities. For this reason the Intensive Neonatal care unit at the regional hospital of Ruse has focused on an early physiotherapy intervention (EPI) program on neonates with hypoxic and ischaemic impairment of the central nervous system (CNS).

Methods The study covers a period of 4 years and includes 172 randomised premature infants of different sexes who were placed in 2 groups (control and experimental). The placement in one of the two groups takes place after a conversation with the parents and informing them about the risk for motor impairments.

Results Hammersmith neurodevelopment and motor development test that was performed to both groups of preterm neonates shows considerable improvement in the motor development to those neonates with EPI immediately after birth. Conclusion Neonate's brain has a high plasticity after birth and great opportunities for recovery. As the EPI improves the condition of neonates at risk of development motor disabilities it can help reduce the cost of disability in the long run.

\section{P0-0625 EFFECTS OF PHOTOTHERAPY ON ANTIOXIDANT STATUS OF PRETERM AND TERM NEONATES WITH JAUNDICE}

${ }^{1} \mathrm{~A}$ Gürol, ${ }^{2} \mathrm{~N}$ Kurt, ${ }^{3} \mathrm{~K}$ Tekgündüz, ${ }^{2} \mathrm{~F}$ Gür, ${ }^{3}$ I Caner, ${ }^{1}$ I Budancamanak, ${ }^{2} \mathrm{~F}$ Polat. ${ }^{1}$ Pediatric Nursing, Atatürk University, Erzurum, Turkey; ${ }^{2}$ Division of Biochemistry, Atatürk University, Erzurum, Turkey; ${ }^{3}$ Division of Neonatology, Atatürk University, Erzurum, Turkey

\subsection{6/archdischild-2014-307384.1267}

Background and aim Phototherapy is generally considered as a safe and well-tolerated therapy in neonatal jaundice. Recently phototherapy has been shown to be related to oxidative stress. In this study we aimed to investigate the effects of phototherapy on antioxidant status of neonates with jaundice.

Methods We conducted a control-grouped pre-test, post-test quasi-experimental design at the newborn unit of a university hospital in Turkey from December 2012 to 2013. Term, very preterm ( 28 to $<32$ weeks), and late preterm ( 32 to $<37$ weeks) newborns hospitalised for significant jaundice requiring phototherapy in the 1-9 days of life were enrolled. A total of 56 newborns were included in this study. The study was approved by 
the local Research Ethics Committee and informed consent was obtained from the parents. Venous blood sampling was performed from a peripheral vein. Levels of malondialdehyde (MDA), superoxide dismutase (SOD), reduced glutathione (GSH), and catalase (CAT) were measured before and $24 \mathrm{~h}$ after phototherapy. Statistical analyses were conducted using the SPSS version 20.0. Statistical significance was accepted at $\mathrm{p}<0.05$.

Results The levels of CAT increased after phototherapy in the all groups; however, this increased was not statistically significant $(\mathrm{p}>0.05)$. The levels of GSH in the very preterm group were also found to be decreased significantly after phototherapy. It was observed that levels of MDA were elevated significantly in term neonates as compared to very preterm and late preterm neonates.

Conclusions Phototherapy did increase the oxidative stress in the term, very preterm, and late preterm newborns with jaundice.

\section{PO-0626 WHICH INFANT MANNEQUIN DO YOU PREFER?: USER PREFERENCE AND BAG MASK ABILITY OF DIFFERENT MANNEQUIN MODELS}

G Hawkes, Y Malik, EM Dempsey, CA Ryan. Department of Paediatrics and Child Health, University College Cork, Cork, Ireland

\subsection{6/archdischild-2014-307384.1268}

Background There are a number of infant mannequins available for teaching newborn and infant bag mask ventilation (BMV) skills. These include the NeoNatalie newborn mannequin and the Baby Anne ${ }^{\mathrm{TM}}$ infant mannequin (both Laerdal Medical Foundation, Stavanger, Norway).

Objectives To examine user preference and user ability to perform effective BMV with these mannequin models.

Methods Each participant was randomised to use the Baby Anne $^{\mathrm{TM}}$ infant mannequin (BA) and the NeoNatalie mannequin fully filled with air (NNA), fully filled with water (NNW) and filled with 50\% air and 50\% water (NNAW), each for a 30 second period. Participants were asked to rate the level of fidelity of the mannequins to a "real baby" on a 5-point likert scale in terms of appearance, weight, feel, tone and realism of ventilation.

Results 20 participants completed this study (10 doctors, 10 nurse/midwives).

Fidelity: BA was similar to NNWA in terms of appearance and similar to NNW in terms of weight. However, in terms of touch, muscle tone and realism of ventilation, NNW and NNWA were similar and greatly exceeded BA and NNA.

Breaths: Although all configurations were generally comparable, NNW had the most effective breaths delivered.

Discussion The NNW and NNAW were both shown to have the highest level of fidelity to a "real baby" and had the highest number of effective ventilations delivered. The NNA was disliked in terms of fidelity, and the most difficult to bag due to the difficulty in positioning the airway. This study suggests that NeoNatalie configurations that contain water are the easiest to provide BMV.

\section{PO-0627 OUTCOMES FOR SUBSTANCE MISUSING WOMEN AND THEIR INFANTS 2006-2011: CHANGES OVER A 5 YEAR TIME PERIOD}

K Johnson, M Balain. Neonatology D Floor Martin Wing, Leeds Teaching Hospital NHS Trust, Leeds West Yorkshire, UK

10.1136/archdischild-2014-307384.1269
Background and aims Substance misuse within the UK population continues to be a public health concern. Many of those using illicit drugs are women of childbearing age.

Infants born to such women are at risk of Neonatal Abstinence Syndrome (NAS) and can require a prolonged stay on the neonatal unit. ${ }^{1}$

Understanding of the demographics and outcomes of this vulnerable group of infants and their mothers is vital in order to evolve services to meet needs and improve outcome.

Methods Retrospective review of substance misusing pregnant women and their offspring, 2006-2011.

Basic demographic data and specific outcome measures for the infants was collected over the 5 year period.

Changes over that 5 year time period were explored.

Results 442 women and their infants were included in the study. All infants were admitted for treatment/observation of NAS.

The majority of women were of white British (85.7\%). Opiates were the most commonly misused substances. $18 \%$ of the babies were low birth weight. Breastfed babies were more likely to be discharged within first 7 days of life compared to artificially fed babies $(47.6 \%$ vs $30.6 \%$, OR 1.55 , $95 \%$ CI 0.95 to 2.53).

Conclusion The management of infants with NAS continues to challenge. Breastfeeding leads to reduced intensity of NAS, and should be recommended to shorten length of hospital stay for infants born to substance misusing mothers.

\section{REFERENCES}

1 Dryden $C$, Young D, Hepburn $M$, et al. Methadone use in pregnancy: factors associated with the development of neonatal abstinence syndrome and implications for healthcare resources. BJOG. 2009;116:655-67

\section{P0-0628 REDUCING SERUM BILIRUBIN DETERMINATIONS IN OUTPATIENT NEWBORNS BY SCREENING WITH TRANSCUTANEOUS BILIRUBINOMETRY}

${ }^{1} \mathrm{R}$ Joseph, ${ }^{2} \mathrm{~A}$ Chinnadurai, ${ }^{3}$ WSY Yeo, ${ }^{4}$ PA Nkouibert, ${ }^{5}$ MS George, ${ }^{4}$ ESY Chan, ${ }^{3}$ WE Tang, ${ }^{6} \mathrm{~J}$ Mathews, ${ }^{2} \mathrm{~J}$ Lee. ${ }^{1}$ Neonatology, National University Hospital and Yong Loo Lin School of Medicine, Singapore, Singapore; ${ }^{2}$ Neonatology, National University Hospital, Singapore, Singapore; ${ }^{3}$ Medicine, NHG Polyclinics, Singapore, Singapore; ${ }^{4}$ Epidemiology, Singapore Clinical Research Institute and Duke-NUS Medical School, Singapore, Singapore; ${ }^{5}$ Neonatology, National University Hospiotal, Singapore, Singapore; ${ }^{6}$ Nursing, NHG Polyclinics, Singapore, Singapore

\subsection{6/archdischild-2014-307384.1270}

Background and aim We have previously shown that using Transcutaneous bilirubin (TCB) values that are $90 \%$ of the age specific SBvalue ( $\mathrm{SBPh}$ ) for phototherapy can markedly reduce the need for invasive serum bilirubin (SB) in Day 1-3 inpatient babies. This study aims to determine if the same principle can be applied in older babies in the outpatient setting.

Methods Three to 14 day olds with jaundice at an outpatient care centre in Singapore and needing a SB measurementwere enrolled after obtaining written informed consent. Their TCB level was determined with a commercial bilirubinometer. Correlations and Bias was determined. Using ROC curves, the TCB values that identified the need for an SB were determined.

Results 1072 paired SB and TCB values were obtained from a Chinese (39\%), Malay (35\%), Indian (14\%) and Others (12\%) cohort. Spearman's correlation coefficientsby age groups ranged from 0.882 (2-5 days) to 0.95 ( $>14$ days). The mean bias (SD) was $-8(23.2)$ and the limits of agreement were -54.4 to 38.4 from the Bland Altman analysis. TCB values that were $90 \%$ of the SBPh yielded accuracy and sensitivity rates (\%) of 90 and 